\title{
Resistance of mitochondrial DNA-depleted cells against oxidized low-density lipoprotein-induced macrophage pyroptosis
}

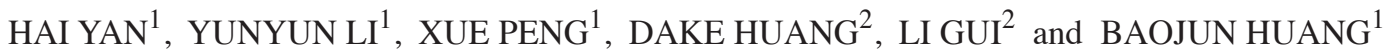 \\ ${ }^{1}$ Department of Immunology; ${ }^{2}$ Comprehensive Laboratory, School of Basic Medical Sciences, \\ Anhui Medical University, Hefei, Anhui 230032, P.R. China
}

Received February 28, 2015; Accepted February 8, 2016

DOI: $10.3892 / \mathrm{mmr} .2016 .5077$

\begin{abstract}
Oxidized low-density lipoprotein (Ox-LDL)-induced macrophage pyroptosis is critical in atherosclerosis inflammation and plaque instability. It has been reported that mitochondrial (mt)DNA-depleted (rho0) cells demonstrate resistance to apoptosis. However, little is known about the susceptibility of rho0 cells to Ox-LDL-induced macrophage pyroptosis. Pyroptosis, a caspase-1-dependent programmed cell death, which compromises membrane integrity, cleaves pro-interleukin (IL)-1 $\beta$ and pro-IL-18 into IL-1 $\beta$ and IL-18, respectively and releases damage-associated molecular pattern molecules, is triggered by a variety of stimuli, including Ox-LDL. In the present study, the expression levels of cleaved caspase-1 and IL-1 $\beta$ in Ox-LDL-treated J774A.1 rho0 cells were observed to be significantly decreased when compared with Ox-LDL-treated J774A.1 normal cells. Furthermore, J774A.1 rho0 cells exhibited a significant reduction in the ratios of dead cells and lactate dehydrogenase release following Ox-LDL stimulation compared with the J774A.1 normal cells. In addition, the loss of mtDNA did not influence Ox-LDL-induced cholesterol accumulation in J774A.1 rho0 cells, which was observed by Oil Red $\mathrm{O}$ staining and CHOD-PAP assay. Finally, J774A.1 rho0 cells exhibited reduced reactive oxygen species (ROS) production and were capable of maintaining the mitochondrial membrane potential following Ox-LDL treatment. Thus, the results indicate that the loss of mtDNA potentially rendered murine macrophage J774A.1 resistant to Ox-LDL-induced pyroptosis by mitigating NACHT, LRR and PYD domains-containing protein 3 inflammasome activation through reducing ROS production. In addition, mtDNA depletion did not interrupt Ox-LDL-induced intracellular lipid accumulation and continued to maintain the mitochondrial membrane potential.
\end{abstract}

Correspondence to: Dr Baojun Huang, Department of Immunology, School of Basic Medical Sciences, Anhui Medical University, 81 Meishan Road, Hefei, Anhui 230032, P.R. China

E-mail: cowboy4314265@qq.com

Key words: macrophage, rho0 cell, pyroptosis, oxidized low-density lipoprotein, reactive oxygen species

\section{Introduction}

As an inflammatory disease of the arteries with characteristic plaque formation, atherosclerosis remains a complicated disease associated with varied risk factors (1). Amongst them, oxidized low-density lipoprotein (Ox-LDL) is key in the pathogenesis of atherosclerosis via the transformation of macrophages and smooth muscle cells into foam cells (2). The intracellular accumulation of Ox-LDL within foam cells activates the NACHT, LRR and PYD domains-containing protein 3 (NLRP3) inflammasome via a possible route of reduced reactive oxygen species (ROS) generation (3) and leads to the release of other damage-associated molecular pattern molecule (DAMP) signals into the extracellular environment. The DAMP signals then further accelerate the deterioration of atherosclerotic plaque.

Numerous studies have described a directly implicating role of the NLRP3 inflammasome in atherosclerosis (3-5). Once activated, the NLRP3 inflammasome cleaves pro-caspase-1 into caspase-1, facilitating further activation of cytokines, interleukin (IL)-1 $\beta$ and IL-18. Pyroptosis, a recently identified form of cell death, has been reported to be directly involved in the non-apoptotic cell death of atherosclerosis (2). Unlike apoptosis, pyroptosis is solely dependent on activation of caspase-1, which will in turn cleave inflammatory cytokines, IL- $1 \beta$ and IL- 18 , into their active forms (6). Recently, Lin et al (2) reported that pyroptosis was the primary form of cell death in Ox-LDL-treated macrophage and atherosclerotic lesions. Yu et al (7) reported that the NLRP3 and absent in melanoma 2 (AIM2) inflammasomes participated in mitochondrial damage in a caspase-1-dependent manner. While activation of the two types of inflammasome may lead to robust ROS generation, the NLRP3 inflammasome exhibits a more potent ability to cause loss of mitochondrial membrane potential (7).

Mitochondrial (mt)DNA-depleted cells, defined as rho0 cells, have become a valuable model for investigating the influence of mitochondria on a variety of cell functions (8-10). Due to their involvement in apoptosis and necrosis, rho0 cells are used to investigate the cell death mechanism (11). Recently, studies have reported that rho0 cells possess a significant resistance to apoptosis that is induced by different agents $(12,13)$. However, to the best of our knowledge, the effects of mtDNA loss on pyroptosis have not yet been elucidated. 
The aim of the present study was to investigate the susceptibility of rho0 cells to Ox-LDL-induced pyroptosis in murine macrophages. Furthermore, the underlying mechanism of rho0 cells mitigating Ox-LDL-induced macrophage pyroptosis was investigated.

\section{Materials and methods}

Cell culture and mtDNA-depleted cells. The J774A.1 murine macrophage cell line was purchased from the China Center for Type Culture Collection (Nanjing, China), and maintained in an atmosphere of $5 \% \mathrm{CO}_{2}$ at $37^{\circ} \mathrm{C}$ in Dulbecco's modified Eagle's medium (DMEM; Thermo Fisher Scientific, Inc., Waltham, MA, USA) containing $10 \%$ fetal calf serum (FCS). The rho0 cell line (J774A.1 rho0) was generated by incubation of J774A.1 in DMEM containing 10\% FCS, $1 \mathrm{mM}$ pyruvate, $50 \mu \mathrm{g} / \mathrm{ml}$ uridine and $50 \mathrm{ng} / \mathrm{ml}$ ethidium bromide (EB), all from Sigma-Aldrich (St. Louis, MO, USA), for 90 days at $37^{\circ} \mathrm{C}$, as described previously (14). Rho0 cell auxotrophy for uridine and pyruvate was assessed by performance of a Cell Counting Kit-8 assay (Guangzhou Yiyuan Biotech Co. Ltd, Guangzhou, China) to confirm the impairment of growth in the absence of these nutrients.

Polymerase chain reaction (PCR). mtDNA from J774A.1 cells (China Center for Type Culture Collection, Wuhan, China) was semi-quantified by PCR to evaluate the remaining mtDNA in the rho0 cells. Total DNA was isolated using DNAzol reagent (Invitrogen; Thermo Fisher Scientific, Inc.) and semiquantified by PCR was performed on a thermal cycler (2720; Thermo Fisher Scientific, Inc.) using PCR Master Mix (2X) (Thermo Fisher Scientific, Inc.) in a final volume of $50 \mu 1$. The conditions for PCR were as follows: $94^{\circ} \mathrm{C}$ for $10 \mathrm{sec}, 25$ cycles at $94^{\circ} \mathrm{C}$ for $45 \mathrm{sec}, 55^{\circ} \mathrm{C}$ for $60 \mathrm{sec}$ and $72^{\circ} \mathrm{C}$ for $45 \mathrm{sec}$. The primer sequences used for PCR were as follows: mtCOI, F 5'-GCCCCAGATATAGCATTCCC-3', R 5'-GTTCATCCT GTTCCTGCTCC-3'. 18S rRNA, F 5'-TAGAGGGACAAG TGGCGTTC-3', R 5'-CGCTGAGCCAGTCAGTGT-3'. All primers were synthesized by Shanghai Sangon Biological Engineering Technology \& Services Co., Ltd. (Shanghai, China). 18s rRNA was used as a refernce gene. The clones containing less mtDNA were then selected and cultured for 12 weeks in the aforementioned medium, but without ethidium bromide.

Western blot analysis. J774A.1 normal and rho0 cells were plated in 6-well plates and grown to 50 60\% confluence. Following stimulation with $100 \mu 1 \mathrm{Ox}-\mathrm{LDL}$, the cells were harvested and lysed using $1 \mathrm{mM}$ phenylmethylsulfonyl fluoride (Sigma-Aldrich). The cell lysates were centrifuged at 14,000 x g for $30 \mathrm{~min}$ at $4^{\circ} \mathrm{C}$. Then the protein concentrations in the supernatants were determined using a bicinchoninic acid kit (Beyotime Institute of Biotechnology, Haimen, China). Proteins (20 mg) from the homogenized samples were separated by $15 \%$ SDS-PAGE for $1 \mathrm{~h}$ at $200 \mathrm{~V}$ and transferred onto polyvinylidene fluoride membranes (EMD Millipore, Boston, MA, USA). After blocking with Tris-buffered saline and Tween-20 (TBST) containing $5 \%$ non-fat milk powder for $2 \mathrm{~h}$ at room temperature, membranes were treated with the following primary antibodies: Rabbit polyclonal anti-pro-caspase 1 (1:500; cat. no. BS1730;
Bioworld Technology, Inc., St Louis Park, MN, USA); rabbit polyclonal anti-cleaved caspase 1 (1:500; cat. no. BS1730; Bioworld Technology, Inc.); rabbit polyclonal anti-IL-1 $\beta$ (1:500; cat. no. BS6067; Bioworld Technology, Inc.) and rabbit polyclonal anti- $\beta$-actin (1:1,000; cat. no. BS-0061R; BIOSS, Beijing, China) at $4^{\circ} \mathrm{C}$ overnight. Next, they were washed with TBST three times and incubated for $1 \mathrm{~h}$ with horseradish peroxidase-conjugated goat anti-rabbit antibody (1:10,000; cat. no. A0208; Beyotime Institute of Biotechnology) at room temperature. Immunoreactivities were detected using an enhanced chemiluminescence detection system (Fast Western Blot kit, ECL substrate; Thermo Fisher Scientific, Inc.) and images were obtained using an automatic digital gel image analysis system (4500SF; Tanon Science \& Technology Co., Ltd., Shanghai, China).

Detection and quantification of lipid aggregation in the macrophages. J774A.1 normal and J774A.1 rho0 cells were seeded in 6-well plates and grown to $50 \sim 60 \%$ confluence. 0 , 25, 50 and $100 \mu \mathrm{g} / \mathrm{ml} \mathrm{Ox-LDL}$ was added to the supernatants for $24 \mathrm{~h}$. The cells were washed with phosphate-buffered saline (PBS) and fixed with formaldehyde. The fixed cells were stained with Oil Red O dye (Nanjing Jiancheng Biotechnology Institute Co., Ltd., Nanjing, China) for $30 \mathrm{~min}$ at $37^{\circ} \mathrm{C}$. The status of intracellular lipid accumulation was detected by microscopy (Nikon Eclipse 80i; Nikon Corporation, Tokyo, Japan) to assess lipid aggregation.

Following stimulation with Ox-LDL, cells were harvested and sonicated on ice for $5 \mathrm{sec}$, with $15 \mathrm{sec}$ intervals using an ultrasonic homogenizer (JY88-IIN; Ningbo Scientz Biotechnology Co., Ltd., Ningbo, China). Following sonication for $10 \mathrm{~min}$, the cells were centrifuged at $8,000 \mathrm{x}$ for $10 \mathrm{~min}$ at $4^{\circ} \mathrm{C}$. The supernatants were then collected and used for total cholesterol quantification using the CHOD-PAP method and a Total Cholesterol Assay kit (Nanjing Jiancheng Biotechnology Institute Co., Ltd.), according to the manufacturer's instructions.

Cell death assay. Cell death was assessed by lactate dehydrogenase $(\mathrm{LDH})$ release (Beyotime Institute of Biotechnology), and EB and acridine orange (AO) staining assays (Sigma-Aldrich).

LDH is a cytosolic enzyme that is released from damaged cells, the presence of which can be detected by the conversion of lactate to pyruvate with a parallel reduction of nicotinamide adenine dinucleotide (NAD) to NADH. For LDH release, $50 \mu 1$ supernatants of J774A.1 normal and rho0 cells following treatment with Ox-LDL were collected and measured using the LDH Cytotoxicity Assay kit (Beyotime Institute of Biotechnology) according to the manufacturer's instructions.

$\mathrm{EB}$ in combination with $\mathrm{AO}(\mathrm{EB} / \mathrm{AO})$ is widely used to evaluate cell death (15). While AO is acidophilic green dye that preferentially stains the lysosomes and nuclei of live cells, EB emits a red fluorescence upon binding to DNA. For EB/AO staining (Sigma-Aldrich), suspended and adherent cells in 96-well plates were collected by centrifugation at $400 \mathrm{x} \mathrm{g}$ for $5 \mathrm{~min}$ and dyed with $8 \mu \mathrm{l} \mathrm{EB/AO} \mathrm{mix} \mathrm{per} \mathrm{well}(50 \mu \mathrm{g} / \mathrm{ml} \mathrm{v} / \mathrm{v})$. Experiments were performed in triplicate and images were acquired using an inverted fluorescence microscopy (Nikon Eclipse 80i; magnification, x400). 
A

$\begin{array}{lcccc}\text { Group } & 1 & 2 & 3 & 4 \\ \text { Ox-LDL } & - & - & + & +\end{array}$

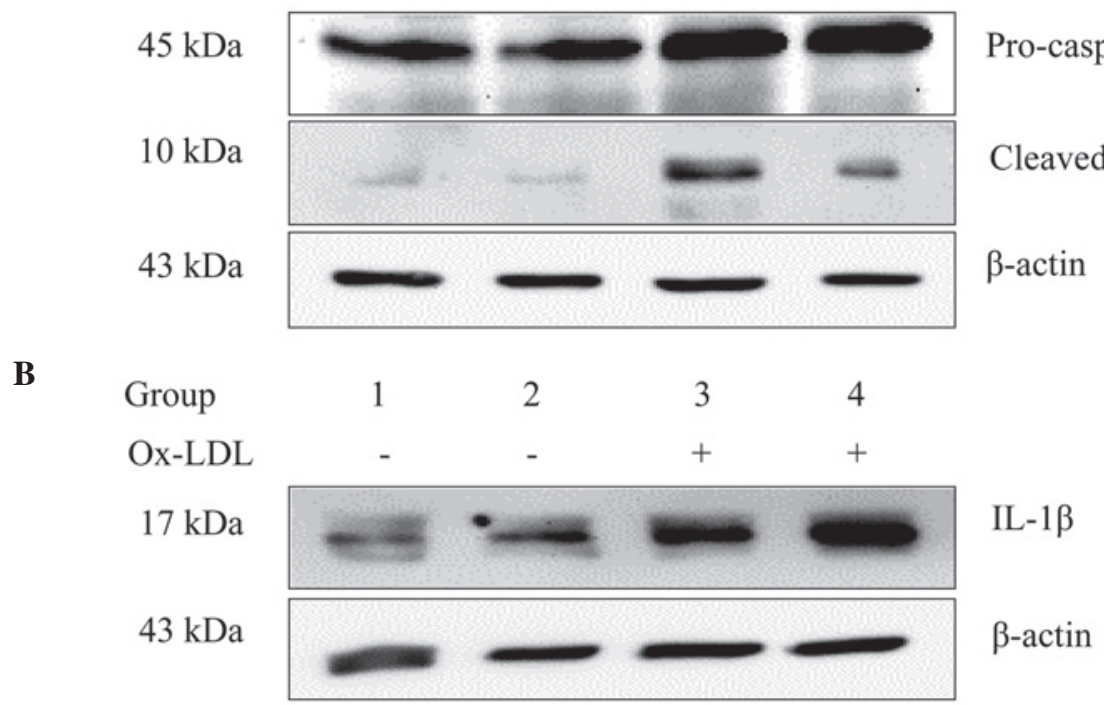

Figure 1. Mitochondrial DNA depletion attenuates Ox-LDL-induced caspase-1 activation and the level of IL-1 $\beta$ expression. J774A.1 rho0 and J774A.1 normal cells were treated with $100 \mu \mathrm{g} / \mathrm{ml}$ Ox-LDL. Following a 24-h incubation, the cells were harvested and western blot analysis was performed. (A) Group 1 and 2 were J774A.1 normal and J774A.1 rho0 cells, respectively that were untreated and served as control groups. Group 3 and 4 were J774A.1 normal and J774A.1 rho0 cells, respectively that were treated with Ox-LDL. (B) Group 1 and 2 were J774A.1 rho0 and J774A.1 normal untreated cell control groups. Group 3 and 4 were J774A.1 rho0 and J774A.1 normal cells treated with Ox-LDL. Ox-LDL, oxidized low-density lipoprotein.

Detection of intracellular ROS production by flow cytometry. The quantity of ROS generated in $100 \mu \mathrm{g} / \mathrm{ml}$ Ox-LDL-treated J774A.1 normal and rho0 cells for $3 \mathrm{~h}$ was measured by fluorescence-activated cell sorting (BD FACSVerse; BD Biosciences, Franklin Lakes, NJ, USA) using fluorescent 2',7'-dichlorodihydrofluorescein diacetate (DCFH-DA; Sigma-Aldrich).

Assessement of mitochondrialmembrane potential by confocal microscopy. Following treatment with $100 \mu \mathrm{g} / \mathrm{ml}$ Ox-LDL for 24 h, J774A.1 normal and rho0 cells were triple-stained with MitoTracker Deep Red, MitoTracker Green and DAPI probes. The change in mitochondrial membrane potential was detected by observing the red signal under a confocal laser scanning microscope (Leica TCS SP5; Leica, Mannheim, Germany).

Statistical analysis. Data are presented as means \pm standard deviation and were analyzed by SPSS 13.0 software (SPSS Inc., Chicago, IL, USA). Statistical analysis was performed using Student's $t$ test for comparison between two groups and $\mathrm{P}<0.05$ was considered to indicate a statistically significant difference.

\section{Results}

mtDNA depletion attenuates Ox-LDL-induced caspase-1 activation and $I L-1 \beta$ expression. Ox-LDLs have been previously shown to trigger caspase-1 activation in human macrophages (2). The effect of mtDNA depletion on Ox-LDL-induced caspase- 1 activation was examined in the present study in murine macrophages. Cleaved caspase-1, which is proteolytically active, promotes the expression of proinflammatory cytokines, such as IL-1 $\beta$ and IL-18 (4). It was found that Ox-LDL stimulation did not increase the expression levels of cleaved caspase- 1 and IL-1 $\beta$ in rho 0 cells. As shown in Fig. 1, cleaved caspase-1 and IL-1 $\beta$ expression levels were markedly reduced in J774A.1 rho0 cells when compared with the J774A.1 normal cell group following treatment with $100 \mu \mathrm{g} / \mathrm{ml}$ Ox-LDL for $24 \mathrm{~h}$.

RhoO cells are not sensitive to $O x$ - LDL-induced macrophage pyroptosis. Rho0 cells have been reported to resist apoptosis (12). Whether mtDNA-depleted cells are resistant to Ox-LDL-induced macrophage pyroptosis was investigated in the present study. As hypothesized, it was found that mtDNA-depleted J774A.1 (rho0) cells exhibited a decreased ratio of dead cells when compared with J774A.1 normal cells following treatment with 50 and $100 \mu \mathrm{g} / \mathrm{ml} \mathrm{Ox-LDL}$ for $24 \mathrm{~h}$ (Fig. 2A-F). Consistent with the results from $\mathrm{EB} / \mathrm{AO}$ staining, a significant reduction in $\mathrm{LDH}$ release was observed in the rho0 cells when compared with the normal J774A.1 cells following treatment with 50 and $100 \mu \mathrm{g} / \mathrm{ml}$ Ox-LDL for $24 \mathrm{~h}$ (Fig. 2G). Together, these results indicate that mtDNA-depleted J774A.1 cells are resistant to Ox-LDL-induced cell death.

Loss of mtDNA does not alter Ox-LDL-induced cholesterol accumulation in macrophages. To investigate whether J774A.1 rho0 cell resistance to ox-LDL-induced cell death was caused by disruption of intracellular cholesterol accumulation, lipid droplets (the existing form of cholesterol in cells) were evaluated by Oil Red $\mathrm{O}$ staining. This indicated no observable difference between J774A.1 rho0 and J774A.1 normal cells (Fig. 3). Further quantification of the intracellular cholesterol results using the CHOD-PAP method indicated that mtDNA depletion did not lead to significant disruption in intracellular total cholesterol following treatment with 0,50 and $100 \mu \mathrm{g} / \mathrm{ml}$ 
A

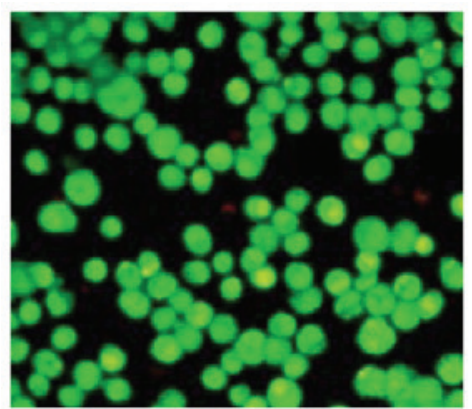

D

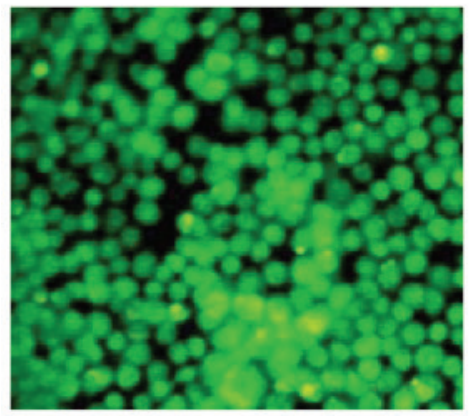

B

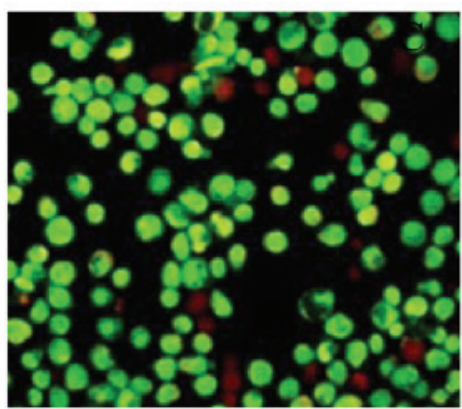

E

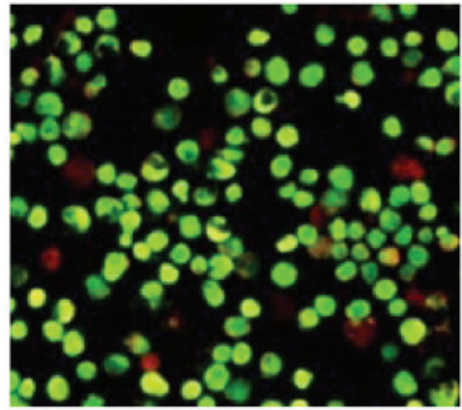

C

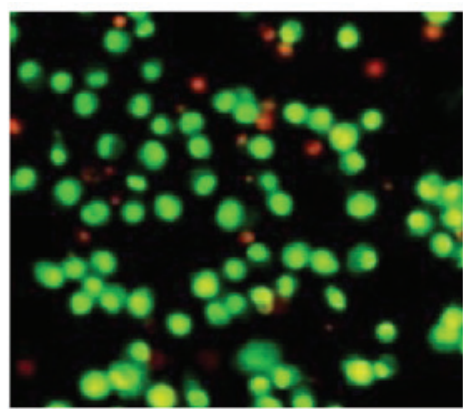

F

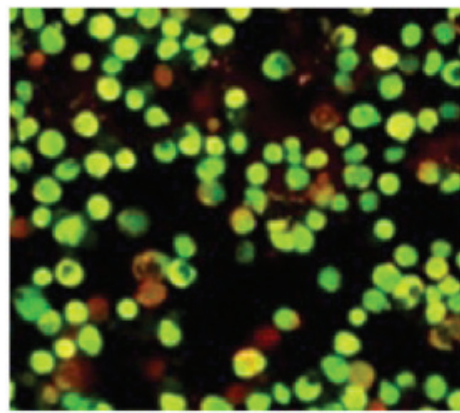

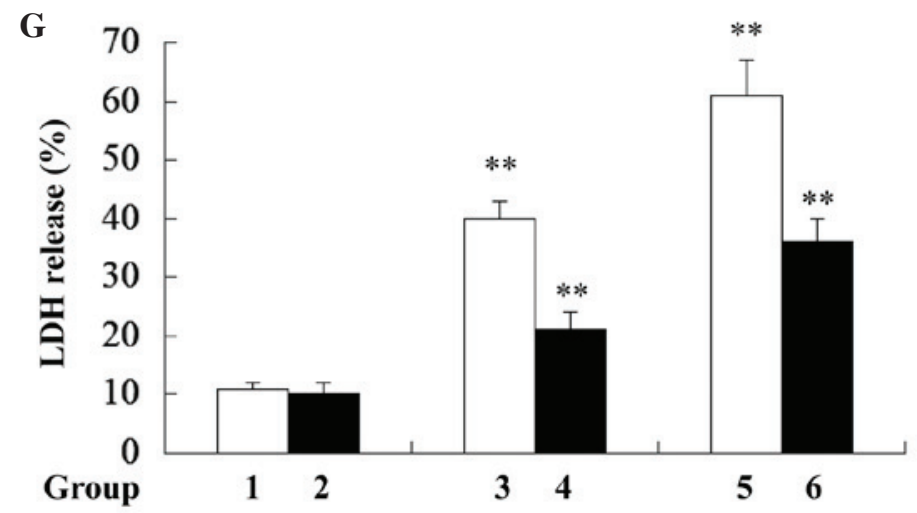

Figure 2. Rho0 cells are not sensitive to Ox-LDL-induced macrophage pyroptosis. (A-C) J774A.1 rho0 and (D-F) normal J774A.1 cells were treated with (B and E) $50 \mu \mathrm{g} / \mathrm{ml}$ or (C and F) $100 \mu \mathrm{g} / \mathrm{ml}$ Ox-LDL for $24 \mathrm{~h}$. Following treatment, cells were stained with ethidium bromide and acridine orange, and observed under an inverted fluorescence microscope. (A and D) Untreated control. Magnification, x400. (G) The supernatant was harvested and an LDH release assay was performed after J774A.1 normal cells (groups 1,3 and 5, white bars) and J774A.1 rho0 cells (groups 2,4 and 6, black bars) were treated with 50 $\mu \mathrm{g} / \mathrm{ml}$ (groups 3 and 4) or $100 \mu \mathrm{g} / \mathrm{ml}$ (groups 5 and 6) Ox-LDL for $24 \mathrm{~h}$. Groups 1 and 2 were the untreated control groups. The data are presented as means \pm standard deviation $(\mathrm{n}=3) .{ }^{* *} \mathrm{P}<0.01$ vs. Ox-LDL group. Ox-LDL, oxidized low-density lipoprotein; LDH, lactate dehydrogenase.

Ox-LDL for $24 \mathrm{~h}$ when the J774A.1 rho0 cells were compared with the J774A.1 normal cells. These results indicate that rho0 cells do not affect Ox-LDL-induced cholesterol accumulation in murine macrophages.

RhoO cells maintain mitochondrial membrane potential and exhibit reduced ROS production following $O x$-LDL treatment. The impact of mtDNA depletion on ROS and mitochondrial membrane potential was determined using MitoTracker Deep Red, MitoTracker Green and DAPI triple staining, and H2DCF-DA probes for ROS labeling with a confocal microscope and flow cytometer, respectively. As shown in Fig. 4A, J774A.1 rho0 cells displayed a similar mitochondrial membrane potential to that of J774A.1 normal cells. Following Ox-LDL treatment, a considerable decrement in mitochondrial membrane potential was observed in the J774A.1 normal cells, but not in the J774A.1 rho0 cells. As shown in Fig. 4B, the J774A.1 normal cells displayed a higher ROS level than that of the J774A.1 rho0 cells following Ox-LDL treatment. These results are consistent with the hypothesis that the mitochondrial respiratory chain is the predominant source of Ox-LDL-induced ROS production in J774A.1 cells, and loss of mtDNA enables J774A.1 rho0 cells to maintain the mitochondrial membrane potential.

\section{Discussion}

In recent years, studies have described the apoptosis resistance of rho0 cells $(12,16)$. Although the exact mechanism remains controversial, the notion that the loss of mtDNA, and its triggering function in apoptotic cell death, leads to oxidant injury and DNA damage (which are exhibited in rho0 cells) is widely held. In an attempt to study the role of mitochondria in pyroptosis, J774A.1 rho0 cells were established by subjecting 
A

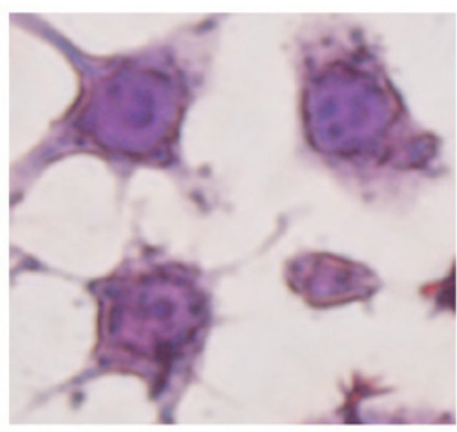

D

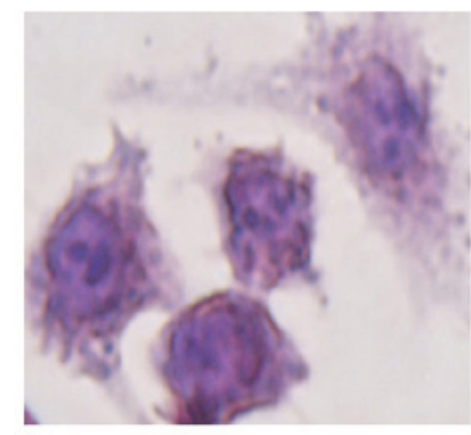

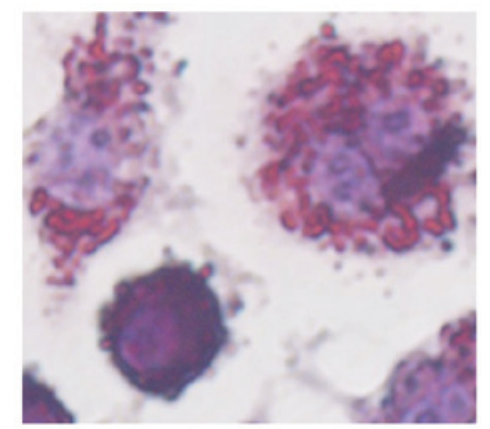

E

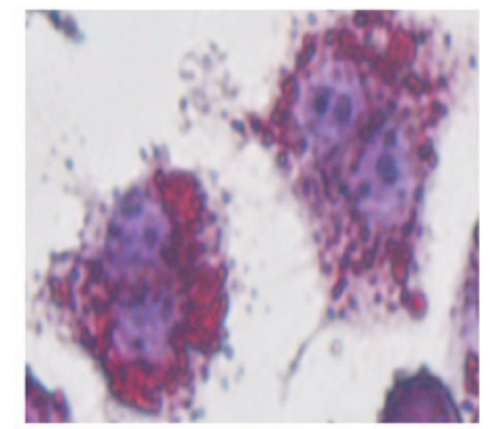

C

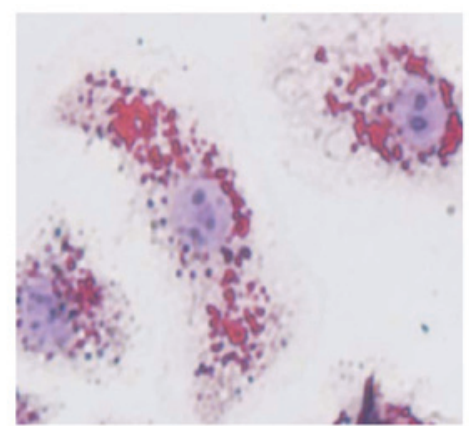

F

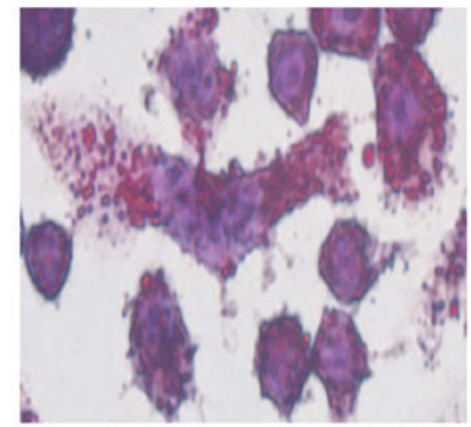

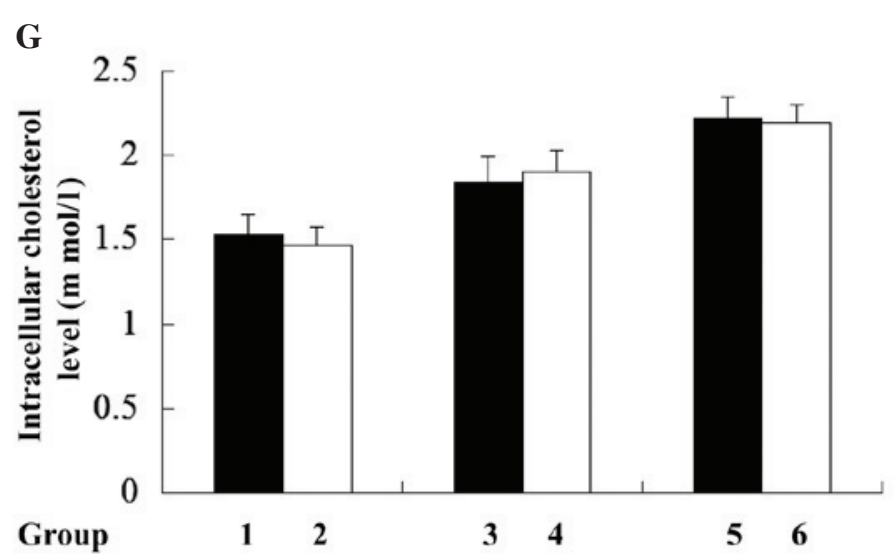

Figure 3. Loss of mitochondrial DNA does not alter Ox-LDL-induced cholesterol accumulation in macrophages. (A-C) J774A.1 rho0 and (D-F) J774A.1 normal cells were treated with (B and E) $50 \mu \mathrm{g} / \mathrm{ml}$ or (C and F) $100 \mu \mathrm{g} / \mathrm{ml} \mathrm{Ox}$-LDL for 24 h. The intracellular lipid droplets were detected by Oil Red O staining. Magnification, x400. (G) Intracellular total cholesterol levels of the two types of cell were quantified using the CHOD-PAP method after J774A.1 rho0 cells (groups 1, 3 and 5) and J774A.1 normal cells (groups 2, 4 and 6) were treated with $50 \mu \mathrm{g} / \mathrm{ml}$ (groups 3 and 4 ) or 100 $\mu$ g/ml (groups 5 and 6) Ox-LDL for $24 \mathrm{~h}$. Group 1 and 2 are untreated control groups. The data are presented as means \pm standard deviation (n=3). Ox-LDL, oxidized low-density lipoprotein.

J774A.1 murine macrophages to chronic EB exposure, according to a previous study (17). The mtDNA-depleted cell was confirmed by PCR and an auxotrophy test (CCK-8; data not shown). In the present study, J774A.1 rho0 cells were reported to be resistant to Ox-LDL-induced macrophage pyroptosis. The results demonstrated that intact, functional mitochondria are essential to the execution of pyroptosis.

Pyroptosis is a recently identified novel form of cell death, which is uniquely depended on caspase-1 activation (18). There exists a number of inflammasomes, which upon activation cleave pro-caspase- 1 into active caspase- 1 and, in turn, initiate the pyroptosis process (19). Among them, the NLRP3 inflammasome has been extensively investigated and identified to be involved in Ox-LDL-induced macrophage pyroptosis in atherosclerosis (20). A number of DAMPs of mitochondrial origin, notably ROS and mtDNA, activate the NLRP3 inflammasome (5). Lin et al (2) demonstrated that Ox-LDL induced macrophage pyroptosis via activation of the NLRP3 inflammasome. Yu et al (7) reported that various inflammasomes, including NLRP3 and AIM2, cause mitochondrial damage and block the mitophagy process via caspase-1 activation. Consistent with Sousa and Soares (21), the results from the present study demonstrated that ROS production was diminished in rho0 cells. However, whether rho0 cells attenuate Ox-LDL-induced macrophage pyroptosis by decreasing ROS production, which subsequently interferes with NLRP3 inflammasome activation, remains to be elucidated. Furthermore, as rho0 cells are devoid of mtDNA, it is also possible that Ox-LDL treatment reduces, or arrests, mtDNA release from mitochondria as a danger signal, which triggers the reduced activation of NLRP3 and AIM2 inflammasomes. Therefore, further investigation is required into the roles of NLRP3 and AIM2 inflammasomes in Ox-LDL-induced rho0 macrophage pyroptosis. 
A
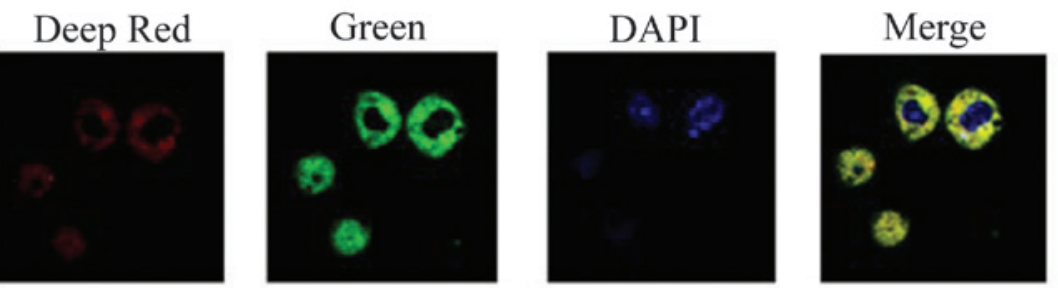

J774A.1
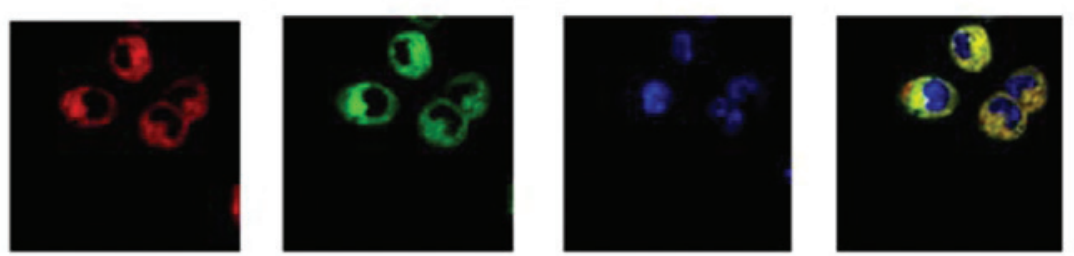

Rho0
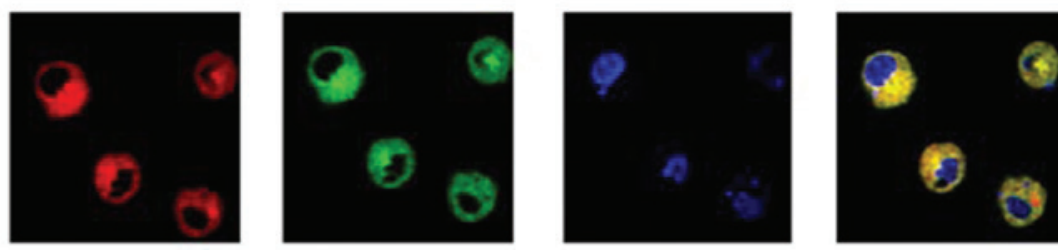

Rho0 ox-LDL
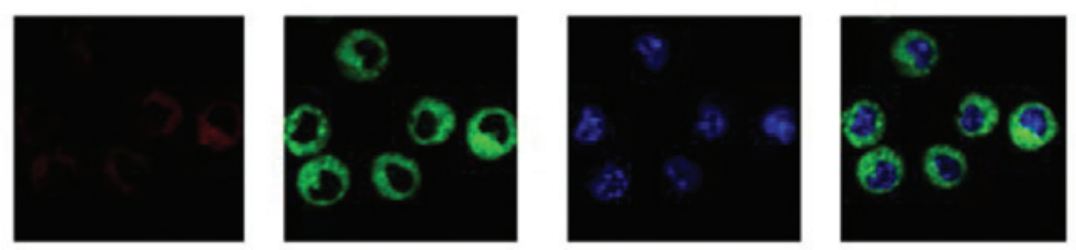

J774A.1 ox-LDL

B
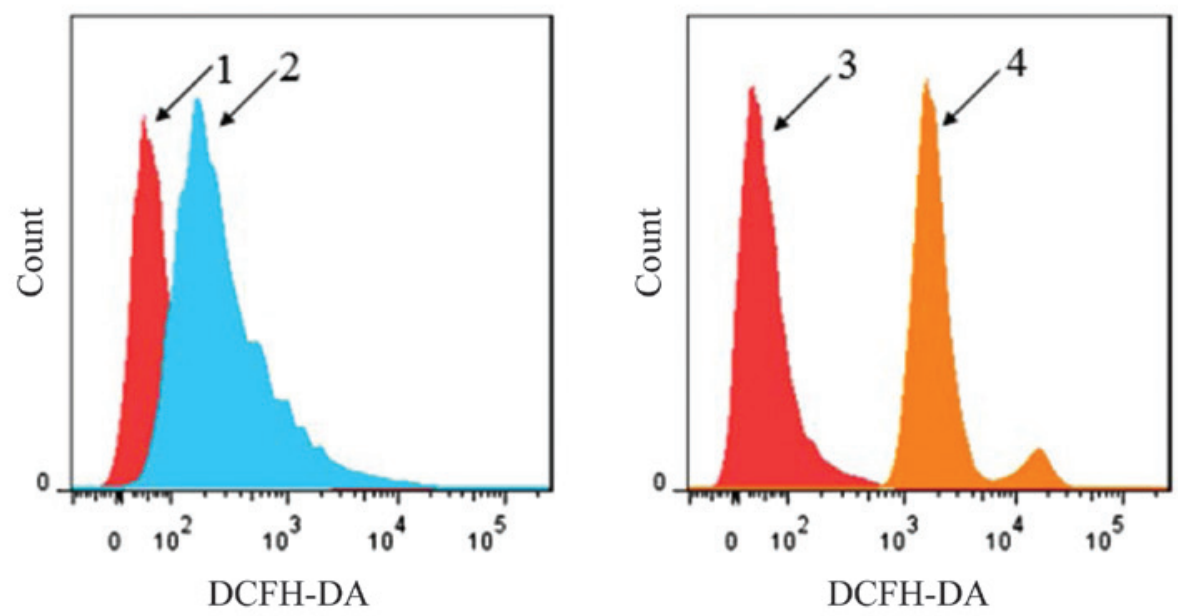

Figure 4. Rho0 cells maintain the mitochondrial membrane potential and exhibit mitigated level of ROS production following Ox-LDL treatment. J774A.1 rho0 and J774A.1 normal cells were treated with $100 \mu \mathrm{g} / \mathrm{ml}$ Ox-LDL. (A) After 24-h incubation, the two types of cell were triple-stained with MitoTracker Deep Red, MitoTracker Green and DAPI, cells were plated on coverslips and observed under a confocal laser scanning microscope. J774A.1 NC and rho0 NC were the untreated control groups. Reduced MitoTracker Deep Red signals indicate loss of mitochondrial membrane potential. (B) Following a $3 \mathrm{~h}$ incubation at $37^{\circ} \mathrm{C}$ with $100 \mu \mathrm{g} / \mathrm{ml}$ Ox-LDL (groups 2 and 4), the J774A.1 rho0 (Group 1 and 2) and J774A.1 normal (groups 3 and 4) cells were harvested and resuspended with DCFH-DA, then incubated for another $30 \mathrm{~min}$. ROS levels were measured via flow cytometry. Groups 1 and 3 are untreated control groups. DCFH-DA, dichloro-dihydro-fluorescein diacetate; ROS, reactive oxygen species; Ox-LDL, oxidized low-density lipoprotein.

Ox-LDL and oxidative stress are closely associated with the pathogenesis of atherosclerosis (2). Previous studies described that lectin-like Ox-LDL receptor 1 (22), toll-like receptor 4 (23) and cluster of differentiation 36 (24) participated in the processes of binding and uptake of Ox-LDL, and foam cell formation. In the current study, J774A.1 rho0 and J774A.1 normal cells were treated with Ox-LDL, and the Oil Red O staining and quantification of intracellular cholesterol results indicated that there was no significant difference in
Ox-LDL-induced lipid accumulation between J774A.1 rho0 and J774A.1 normal cells. The results indicate that cells continue uptake of Ox-LDL and form intracellular lipid droplets without functional mitochondria.

In conclusion, the present study demonstrates that J774A.1 rho0 cells are resistant to Ox-LDL-induced pyroptosis, potentially via decreased ROS production and, thus, a weaker activation of the NLRP3 inflammasome. In addition, the current data implied that the loss of mtDNA did not affect 
Ox-LDL-induced intracellular lipid accumulation and mitochondrial membrane potential. However, the exact mechanism of rho0 cell resistance to pyroptosis remains unclear and requires further investigation.

\section{Acknowledgements}

The present study was supported by National Natural Science Foundation of China Grants (grant no. 30972791) awarded to Baojun Huang.

\section{References}

1. Conti P and Shaik-Dasthagirisaeb Y: Atherosclerosis: A chronic inflammatory disease mediated by mast cells. Cent Eur J Immunol 40: 380-386, 2015.

2. Lin J, Shou X, Mao X, Dong J, Mohabeer N, Kushwaha KK, Wang L, Su Y, Fang H and Li D: Oxidized low density lipoprotein induced caspase-1 mediated pyroptotic cell death in macrophages: Implication in lesion instability? PLoS One 8: e62148, 2013.

3. Rajamäki K, Lappalainen J, Oörni K, Välimäki E, Matikainen S, Kovanen PT and Eklund KK: Cholesterol crystals activate the NLRP3 inflammasome in human macrophages: A novel link between cholesterol metabolism and inflammation. PLoS One 5: e11765, 2010

4. Jiang Y, Wang M, Huang K, Zhang Z, Shao N, Zhang Y, Wang W and Wang S: Oxidized low-density lipoprotein induces secretion of interleukin-1 $\beta$ by macrophages via reactive oxygen species-dependent NLRP3 inflammasome activation. Biochem Biophys Res Commun 425: 121-126, 2012.

5. Zhou R, Tardivel A, Thorens B, Choi I and Tschopp J: Thioredoxin-interacting protein links oxidative stress to inflammasome activation. Nat Immunol 11: 136-140, 2010

6. Dostert C, Pétrilli V, Van Bruggen R, Steele C, Mossman BT and Tschopp J: Innate immune activation through Nalp3 inflammasome sensing of asbestos and silica. Science 320: 674-677, 2008.

7. Yu J, Nagasu H, Murakami T, Hoang H, Broderick L, Hoffman HM and Horng T: Inflammasome activation leads to Caspase-1-dependent mitochondrial damage and block of mitophagy. Proc Natl Acad Sci USA 111: 15514-15519, 2014.

8. Park SY, Choi B, Cheon H, Pak YK, Kulawiec M, Singh KK and Lee MS: Cellular aging of mitochondrial DNA-depleted cells. Biochem Biophys Res Commun 325: 1399-1405, 2004.

9. Arduino DM, Esteves AR, Cortes L, Silva DF, Patel B, Grazina M, Swerdlow RH, Oliveira CR and Cardoso SM: Mitochondrial metabolism in Parkinson's disease impairs quality control autophagy by hampering microtubule-dependent traffic. Hum Mol Genet 21: 4680-4702, 2012
10. Prigione A and Cortopassi G: Mitochondrial DNA deletions and chloramphenicol treatment stimulate the autophagic transcript ATG12. Autophagy 3: 377-380, 2007.

11. Park SY, Chang I, Kim JY, Kang SW, Park SH, Singh K and Lee MS: Resistance of mitochondrial DNA-depleted cells against cell death: Role of mitochondrial superoxide dismutase. J Biol Chem 279: 7512-7520, 2004.

12. Ferraresi R, Troiano L, Pinti M, Roat E, Lugli E, Quaglino D, Taverna D, Bellizzi D, Passarino G and Cossarizza A: Resistance of mtDNA-depleted cells to apoptosis. Cytometry A 73: 528-537, 2008.

13. Brar SS, Meyer JN, Bortner CD, Van Houten B and Martin WJ II: Mitochondrial DNA-depleted A549 cells are resistant to bleomycin. Am J Physiol Lung Cell Mol Physiol 303: L413-L424, 2012.

14. Kirii H, Niwa T, Yamada Y, Wada H, Saito K, Iwakura Y, Asano M, Moriwaki H and Seishima M: Lack of interleukin-1beta decreases the severity of atherosclerosis in ApoE-deficient mice. Arterioscler Thromb Vasc Biol 23: 656-660, 2003

15. Ribble D, Goldstein NB, Norris DA and Shellman YG: A simple technique for quantifying apoptosis in 96-well plates. BMC Biotechnol 5: 12, 2005.

16. Lee MS, Kim JY and Park SY: Resistance of rho(0) cells against apoptosis. Ann N Y Acad Sci 1011: 146-153, 2004.

17. King MP and Attardi G: Isolation of human cell lines lacking mitochondrial DNA. Methods Enzymol 264: 304-313, 1996.

18. Bergsbaken T, Fink SL and Cookson BT: Pyroptosis: Host cell death and inflammation. Nat Rev Microbiol 7: 99-109, 2009.

19. Fink SL and Cookson BT: Apoptosis, pyroptosis and necrosis: Mechanistic description of dead and dying eukaryotic cells. Infect Immun 73: 1907-1916, 2005.

20. Zhou R, Yazdi AS, Menu P and Tschopp J: A role for mitochondria in NLRP3 inflammasome activation. Nature 469: 221-225, 2011

21. Sousa CA and Soares EV: Mitochondria are the main source and one of the targets of $\mathrm{Pb}$ (lead)-induced oxidative stress in the yeast Saccharomyces cerevisiae. Appl Microbiol Biotechnol 98: 5153-5160, 2014.

22. Nowicki M, Müller K, Serke H, Kosacka J, Vilser C, Ricken A and Spanel-Borowski K: Oxidized low-density lipoprotein (oxLDL)-induced cell death in dorsal root ganglion cell cultures depends not on the lectin-like oxLDL receptor-1 but on the toll-like receptor-4. J Neurosci Res 88: 403-412, 2010.

23. Serke H, Vilser C, Nowicki M, Hmeidan FA, Blumenauer V, Hummitzsch K, Lösche A and Spanel-Borowski K: Granulosa cell subtypes respond by autophagy or cell death to oxLDL-dependent activation of the oxidized lipoprotein receptor 1 and toll-like 4 receptor. Autophagy 5: 991-1003, 2009.

24. Serke H, Bausenwein J, Hirrlinger J, Nowicki M, Vilser C, Jogschies P, Hmeidan FA, Blumenauer V and Spanel-Borowski K: Granulosa cell subtypes vary in response to oxidized low-density lipoprotein as regards specific lipoprotein receptors and antioxidant enzyme activity. J Clin Endocrinol Metab 95: 3480-3490, 2010. 